Tạp chí Các Khoa học về Trái Đất, 38 (1), 79-89

Viện Hàn lâm Khoa học và Công nghệ Việt Nam
Tạp chí Các Khoa học về Trái Đất
(VAST)
Website: http://www.vjs.ac.vn/index.php/jse

\title{
Nghiên cứu phân vùng chất lượng nước dưới đất theo độ tổng khoáng hóa tỉnh Hưng Yên
}

\author{
Nguyễn Đức Rỡi \\ Viện Địa chất, Viện Hàn lâm Khoa học và Công nghệ Việt Nam
}

Chấp nhận đăng: 12 - 2 - 2016

ABSTRACT

Groundwater quality zonation by total dissolved solids for Hung Yen province

Hưng Yên province has relatively abudant groundwater $(\mathrm{GW})$ resource, howerver the groundwater quality distribution is variable, including total dissolved solids (TDS).

Groundwater zonation by TDS is important for sustainable GW planning in term of quality. Analysis of the TDS spatial distribution for all the three aquifers Holocene (qh), upper Pleistocene (qp2) and lower Pleistocene (qp1) have given variogram of a power type of very high correlation coefficient with the actual data which means a high reliability. TDS kriging had been applied to compile GW zonation by TDS contents in three levels: fresh, light brackish and brackish for the aquigfers qh, qp2 and qp1. The areas of GW of light brackish to brackish area: (i) aquigfer qh: $\sim 310 \mathrm{~km}^{2}\left(\sim 33 \%\right.$ of the area); (ii) aquigfer qp2: $\sim 10 \mathrm{~km}^{2}$ ( $\sim 23 \%$ of the area); (iii) aquigfer qp1: $\sim 180 \mathrm{~km}^{2}(\sim 20 \%$ of the area). The areas of brackish $\mathrm{GW}$ of qh and qp2 are very different, which means the two aquifers are of insignificant vertical interaction or no interaction thanks to the aquitard inbetween. The areas of brackish GW of qp2 and qp1 are relatively similar with only lightly difference in shapes, which may be an evidence of good hydraulic interaction between the two aquifer there Based on the combination of GW zonation by TDS and the locations and abstraction quantities of existing GW abstraction facilities, some salinization prevention measures, inluding artificial recharge had been proposed.

Keywords: Holocene, Pleistocene, Groundwater, TDS, Salty water, Brackish water, Variogram, Kriging.

(C)2016 Vietnam Academy of Science and Technology

\section{Mở đầu}

Tỉnh Hưng Yên có vị trí địa lý nằm ở trung tâm vùng đồng bằng Sông Hồng, là vùng kinh tế trọng điểm của vùng đồng bằng Bắc Bộ và tam giác kinh tế Hà Nội - Hải Phòng - Quảng Ninh.

Do sự phát triển kinh tế xã hội mạnh mẽ cùng với việc xây dựng và phát triển nhanh chóng các nhà máy, xí nghiệp, các khu công nghiệp $(\mathrm{KCN})$, các khu đô thị mới,... nên nhu cầu khai thác sử dụng nước dưới đất $(\mathrm{NDĐ})$ trong khu vực ngày

Email: roivdc@gmail.com càng tăng. Trên địa bàn tỉnh hiện có nhiều công trình khai thác NDĐ tập trung phục vụ các KCN, các khu đô thị, các vùng nông thôn và hàng trăm nghìn giếng khoan đường kính nhỏ (dạng Unicef) khai thác NDĐ quy mô hộ gia đình. Tổng lưu lượng khai thác NDĐ hiện nay trên địa bàn tỉnh là khoảng $267.000 \mathrm{~m}^{3} / \mathrm{ng}$ (Sở Tài nguyên và Môi trường Hưng Yên, 2014). Dự kiến nhu cầu sử dụng nước trên địa bàn tỉnh đến năm 2020 khoảng $468.000 \mathrm{~m}^{3} / \mathrm{ng}$, trong đó NDÐ khoảng $456.000 \mathrm{~m}^{3} / \mathrm{ng}$. Ngoài ra, các vùng lân cận với tỉnh còn có nhà máy nước Cẩm Giàng - Hải Dương đang khai thác với lưu lượng $10.200 \mathrm{~m}^{3} / \mathrm{ng}$, 


\section{N.Đ. Rỡi/Tạp chí Các Khoa học về Trái Đất, Tập 38 (2016)}

nhà máy nước Gia Lâm - Hà Nội khai thác $60.000 \mathrm{~m}^{3} / \mathrm{ng}$ và các giếng khoan $\mathrm{DDT}, \mathrm{Z112}$, HVHC, GCĐ, Hanel, GTD2620 thuộc địa bàn huyện Gia Lâm đang khai thác với tổng lưu lượng khoảng trên $14.000 \mathrm{~m}^{3} / \mathrm{ng}$ (Phạm Quý Nhân và nnk, 2007).

Trong khi đó chất lượng NDĐ rất khác nhau, trong đó nổi bật nhất là sự có mặt của các diện tích NDĐ có độ tổng khoáng hóa $>1 \mathrm{~g} / 1$ (được xem là ranh giới mặn - nhạt trong địa chất thủy văn). Xác định được phân bố chất lượng NDĐ trong khu vực sẽ là cơ sở quan trọng và cần thiết cho công tác qui hoạch và quản lý khai thác NDĐ bền vững về mặt chất lượng. Bài viết trình bày các kết quả nghiên cứu về phân vùng chất lượng NDĐ theo độ tổng khoáng hóa theo không gian làm cơ sở khoa học đề xuất các biện pháp quy hoạch khai thác phát triển NDĐ (bảo vệ và cải thiện chất lượng,...) trong khu vực nghiên cứu.

\section{Cơ sở tài liệu và phương pháp sử dụng}

\subsection{Cơ sở tài liệu}

Tài liệu về chất lượng NDĐ sử dụng trong bài viết này là các tài liệu của các đề tài do tác giả làm chủ nhiệm: Đề tài "Đánh giá tổng hợp, quy hoạch sử dụng NDĐ tỉnh Hưng Yên đến năm 2020" thực hiện năm 2009 và đề tài "Điều tra, xây dựng cơ sở dữ liệu tài nguyên NDĐ, thành lập bản đồ địa chất thủy văn tỉ lệ 1:50.000 tỉnh Hưng Yên phục vụ quản lý khai thác, sử dụng hợp lý tài nguyên NDĐ" thực hiện năm 2011 do Viện Địa chất chủ trì và do Sở Tài nguyên và Môi trường Hưng Yên quản lý. Ngoài ra là các tài liệu tham khảo của các đề án khác như: "Đề án đo vẽ lập bản đồ địa chất, địa chất thủy văn - địa chất công trình tỷ lệ 1:50.000 vùng Hưng Yên - Phủ Lý" do Nguyễn Văn Độ và nnk, 2002 thuộc Liên đoàn Quy hoạch và Điều tra Tài nguyên nước Miền Bắc thuộc Bộ TN\&MT thực hiện năm 2002; "Đề án thăm dò đánh giá trữ lượng khai thác NDĐ tại Trung tâm dệt kim Phố Nối công suất $5.000 \mathrm{~m}^{3} / \mathrm{ng}^{\prime \prime}$ do Phạm Quý Nhân và nnk thuộc Công ty Tư vấn Triển khai công nghệ và xây dựng Mỏ - Địa chất thực hiện năm $2007, \ldots$

\section{2. Độ tổng khoáng hóa}

Độ tổng khoáng hóa của NDĐ có thể được xác định trực tiếp bằng việc phân tích các thành phần hóa học cơ bản của mẫu NDĐ hoặc đối với nước có hàm lượng tổng khoáng hóa chủ yếu là muối ăn thì xác định gần đúng qua hàm lượng muối ăn (thông qua hàm lượng cation $\mathrm{Na}^{+}$và anion $\mathrm{Cl}^{-}$) hoặc xác định gần đúng thông qua tương quan toán học được xác lập qua quan hệ giữa độ dẫn điện và độ tổng khoáng hóa (được xác định bằng phân tích hóa học). Trong khuôn khổ bài viết này, với số lượng lớn các số liệu độ dẫn điện của NDĐ được đo đạc tại thực địa, độ tổng khoáng hóa được xác định thông qua tương quan giữa độ tổng khoáng hóa và độ dẫn điện của nước.

Độ tổng khoáng hóa $(\mathrm{M})$ của nước nói chung và $\mathrm{NDĐ}$ nói riêng có thể xác định thông qua độ dẫn điện của nước $(\mathrm{EC})$ (thông thường đo bằng máy đo độ dẫn điện của nước) theo công thức tổng quát:

$$
M(\mathrm{~g} / \mathrm{l})=a \times E C(\mathrm{mS} / \mathrm{cm})
$$

Trong đó, $a$ là hệ số phụ thuộc loại nước và trị số $\mathrm{EC}$, được xác định như sau: đối với NDĐ không thuộc loại siêu nhạt, đồng thời cũng không thuộc loại có $M$ quá lớn với $\mathrm{EC}<5 \mathrm{mS} / \mathrm{cm}$ thì a lây trong khoảng $0,5 \div 0,735$ (trung bình 0,65 ); đối với nước siêu nhạt a trong khoảng $0,47 \div 0,5$ và khi $E C$ $>5 \mathrm{mS} / \mathrm{cm}$ thì a $0,735 \div 0,8$ (Kemker, 2014).

NDĐ khu vực nghiên cứu không thuộc loại nước siêu nhạt và theo các kết quả đo $\mathrm{EC}$ luôn $<5$ $\mathrm{mS} / \mathrm{cm}$, nên để tính toán $M$ của NDĐ, sử dụng hệ số $\mathrm{a}=0,65$.

\subsection{Tổng quan các chỉ tiêu khác về chất lự̣ng NDÐ}

Các chỉ tiêu khác về chất lượng NDĐ đã được nghiên cứu bao gồm độ cứng tổng cộng, tổng các chất hữu cơ (thông qua nhu cầu ô xy hóa học COD), $\mathrm{SO}_{4}, \mathrm{NH}_{4}, \mathrm{NO}_{2}, \mathrm{NO}_{3}$, Phenol, $\mathrm{Mn}, \mathrm{Fe}, \mathrm{Cu}$, $\mathrm{Pb}, \mathrm{Zn}, \mathrm{Cd}, \mathrm{Cr}, \mathrm{Hg}$, As, Ecoli và Coliform. Tuy nhiên số lượng mẫu không được nhiều như số lượng mẫu về $M$, trung bình đối với Tầng chứa nước (TCN) qh là $57,7 \mathrm{~km}^{2} / 1$ mẫu, TCN qp2: $13,4 \mathrm{~km}^{2} / 1$ mẫu và TCN qp $1: 22,5 \mathrm{~km}^{2} / 1$ mẫu.

Tất cả các mẫu nước phân tích của cả $03 \mathrm{TCN}$ có hàm lượng $\mathrm{COD}, \mathrm{SO}_{4}, \mathrm{NO}_{3}, \mathrm{Cu}, \mathrm{Zn}, \mathrm{Cd}, \mathrm{Cr}$ thỏa mãn QCVN 09:2008 (Bộ Tài nguyên và Môi trường, 2008). Các chỉ tiêu chất lượng gồm tổng các chất hữu cơ, $\mathrm{NH}_{4}, \mathrm{Mn}, \mathrm{Fe}$, Ecoli, Coliform có hàm lượng không thỏa mãn QCVN 09:2008 với tỷ lệ khoảng $30 \%$ trở lên. Trong khuôn khổ bài viết này sẽ không đi sâu đánh giá các chỉ tiêu chất lượng này, mà chỉ tập trung vào độ tổng khoáng hóa của nước. 
Tạp chí Các Khoa học về Trái Đất, 38 (1), 79-89

Số lượng và phần trăm mẫu có các chỉ tiêu trí lấy mẫu nước phân tích các chỉ tiêu khác về không thỏa mãn QCVN 09:2008 được tổng hợp chất lượng NDĐ của các TCN thể hiện trong trong bảng 1 ; vị trí đo độ dẫn điện của NDĐ và vị $\quad$ hình 1 .

\begin{tabular}{|c|c|c|c|c|c|c|c|c|c|c|c|c|c|}
\hline Tầng & Số mẫu & Độ cứng & COD & NH4 & $\mathrm{NO} 2$ & Phenol & $\mathrm{Mn}$ & $\mathrm{Fe}$ & $\mathrm{Pb}$ & $\mathrm{Hg}$ & As & Ecoli & Coli-form \\
\hline$\overline{\text { QCVN 09:2008 }}$ & & 500 & 4 & 0,1 & 1 & 0,001 & 0,5 & 5 & 0,01 & 0,001 & 0,05 & 0 & 3 \\
\hline $\mathrm{qh}$ & 16 & 0 & 1 & 5 & 0 & 0 & 7 & 5 & 0 & 2 & 0 & 8 & 8 \\
\hline & $\%$ & 0 & 6,5 & 31,3 & 0 & 0 & 43,8 & 31,3 & 0 & 12,5 & 0 & 50 & 50 \\
\hline qp2 & 69 & 2 & 27 & 20 & 16 & 8 & 39 & 31 & 1 & 5 & 0 & 24 & 23 \\
\hline & $\%$ & 2,9 & 39,1 & 29,0 & 23,2 & 11,6 & 56,5 & 44,9 & 1,4 & 7,2 & 0 & 34,8 & 33,3 \\
\hline $\mathrm{qp} 1$ & 41 & 0 & 15 & 17 & 1 & 5 & 21 & 21 & 1 & 2 & 2 & 14 & 17 \\
\hline
\end{tabular}

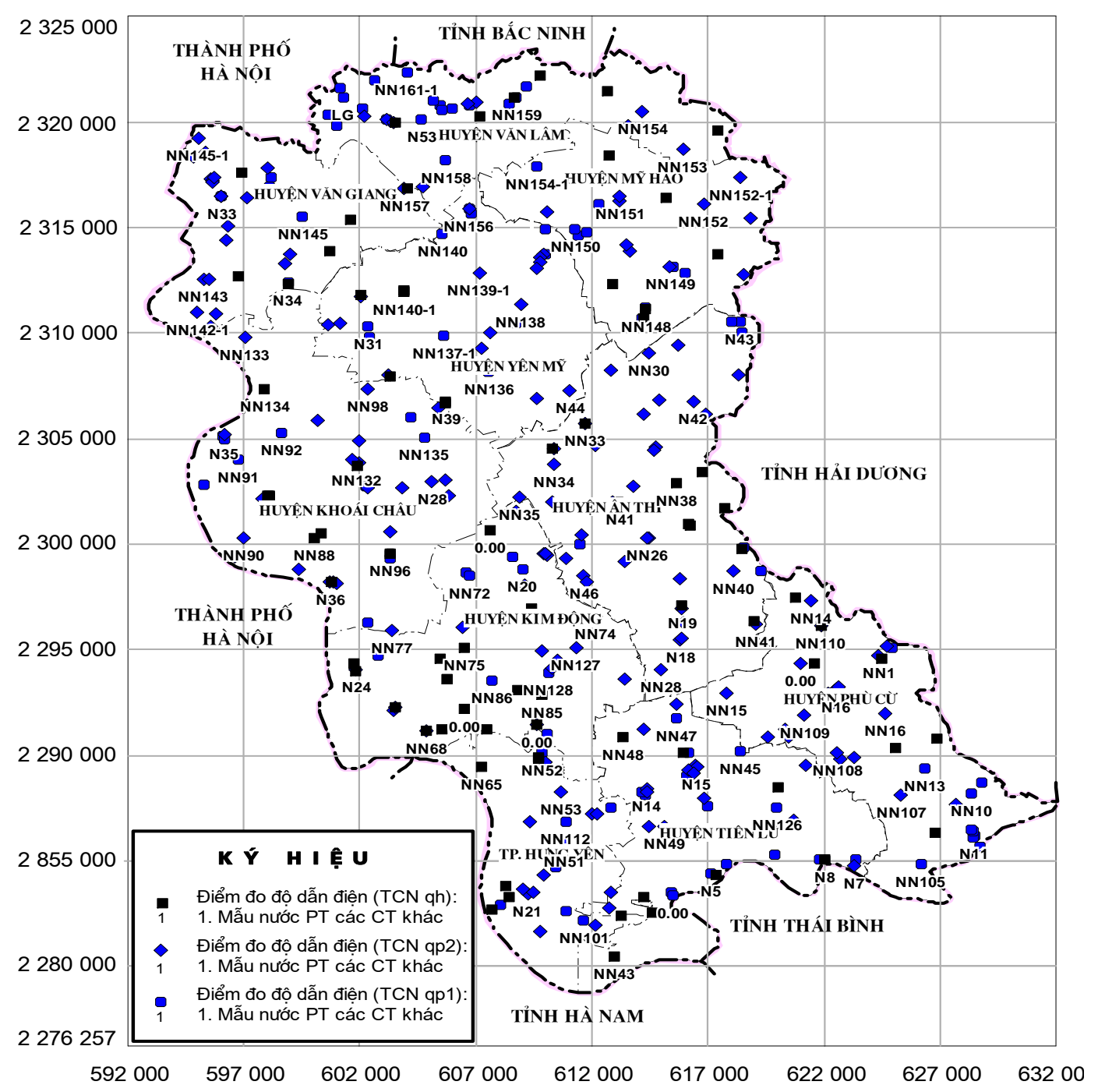

Hình 1. Sơ đồ vị trí các điểm đo độ dẫn điện của NDĐ và vị trí lấy mẫu phân tích các chỉ tiêu khác về chất lượng của NDĐ 


\section{N.Đ. Rỡi/Tạp chí Các Khoa học về Trái Đất, Tập 38 (2016)}

\subsection{Phương pháp phân tích không gian sử dụng phân vùng chất lự̛̣n $\mathrm{NDP}$}

Trong thực tế điều tra tài nguyên NDĐ, các thông số chất lượng NDĐ thường chỉ được xác định tại một số điểm nhất định trong không gian. Trong khi đó xác định sự phân bố chất lượng NDĐ trên toàn bộ miền phân bố của $\mathrm{TCN}$ là quan trọng và cần thiết. Các giá trị các thông số chất lượng NDĐ phần lớn là dạng biến ngẫu nhiên, mà trong xác suất thống kê chúng luôn tuân thủ một quy luật phân bố nhất định nào đó. Chính vì vậy mà một phương pháp mang tên "Địa Kriging" đã được xây dựng nhằm xác định ước lượng giá trị các thông số tại các vị trí mong muốn dựa trên các giá trị đã biết của thông số tại một số vị trí xác định thông qua việc xác định qui luật phân bố của các thông số này trong không gian.

Phương pháp này lý giải như sau (Ghislain de Marsily, 1987): Giá trị $Z(\mathbf{x})$ của thông số đang nghiên cứu là một hàm ngẫu nhiên $Z(\mathbf{x}, \xi)$ chỉ có một phép thể hiện; $\mathbf{x}$ là tọa độ không gian (chẳng hạn tọa độ trong không gian một chiều $x$, hai chiều $x y$ hoặc ba chiều $x y z)$; $\xi$ là biến trạng thái trong không gian của các phép thể hiện (chẳng hạn $Z\left(x, \xi_{1}\right)$ là phép thể hiện của $\left.\xi_{1}\right) ; Z\left(\mathbf{x}_{0}, \xi\right)$ là biến ngẫu nhiên và là một tập hợp các phép thể hiện của hàm ngẫu nhiên $Z(\mathbf{x}, \xi)$ tại điểm $\mathbf{x}_{0} ; E\left[Z\left(\mathbf{x}, \xi_{1}\right)\right]$ là kỳ vọng toán học của $Z\left(\mathbf{x}, \xi_{1}\right)$; và hiệp phương sai là:

$$
\begin{aligned}
& \operatorname{cov}\left(\mathbf{x}_{1}, \mathbf{x}_{2}\right)=E\left\{\left[Z\left(\mathbf{x}_{1}, \xi\right)-Z(\mathbf{x}, \xi)\right]\right. \\
& \left.\left[Z\left(\mathbf{x}_{2}, \xi\right)-Z(\mathbf{x}, \xi)\right]\right\}=C(\mathbf{h})
\end{aligned}
$$

trong đó $\mathbf{h} \equiv \mathbf{x}_{1}-\mathbf{x}_{2}$ là khoảng cách giữa hai điểm $\mathbf{x}_{1}$ và $\mathbf{x}_{2}$.

Địa Kriging bao gồm hai bước: (i) xác định cấu trúc của hàm ngẫu nhiên $Z\left(\mathbf{x}_{i}, \xi\right)$ dựa trên các phép thể hiện thí nghiệm (thông số xác định qua thí nghiệm) $Z\left(\mathbf{x}_{i}, \xi_{1}\right)$; và (ii) xác định $Z\left(x_{0}, \xi_{1}\right)$ (hoặc thông số $Y$ bất kỳ) tại điểm $\mathbf{x}_{0}$ bất kỳ bằng phép tính toán tối ưu qua phép toán có trọng số như sau:

$$
Y_{0}^{*}=Y^{*}\left(\mathbf{x}_{0}\right)=\sum_{i=1}^{n} \lambda_{0}^{i} Y_{i}
$$

trong đó: $\lambda_{0}^{i}$ - là các hệ số trọng số ứng với điểm đã đo $i$ đối với điểm tính toán 0 .

Đáng lưu ý là chúng ta đang cho thông số là hàm ngẫu nhiên, nên các giá trị xác định được bằng thí nghiệm chỉ là một trong các phép thể hiện mà thôi (chẳng hạn phép thể hiện $\xi_{1}$, và do đó giá trị tính toán được cũng tương ứng với phép thể hiện $\xi_{1}$ ).

Thông thường phân ra ba trường hợp Địa Kriging: (i) giả thiết dừng; (ii) giả thiết nội tại (số gia dừng); (iii) giả thiết không dừng.

\subsubsection{Giả thiết dìng}

Hàm ngẫu nhiên của giả thiết này gọi là dừng bậc hai nếu kỳ vọng toán học của nó là hằng số và hàm hiệp phương sai chỉ phụ thuộc vào khoảng cách. Trong trường hợp này ta có:

$$
\begin{aligned}
& E[Z(\mathbf{x}, \xi)]=m ; \operatorname{cov}\left(\mathbf{x}_{1}, \mathbf{x}_{2}\right)=C(\mathbf{h}) ; \\
& C(0)=\operatorname{var}(Z)=\sigma_{Z}^{2}
\end{aligned}
$$

trong đó: $m$ - giá trị trung bình đã biết, $C(\mathbf{h})$ - hàm hiệp phương sai quyết định cấu trúc của thông số (hình 2 ), và $\sigma_{Z}^{2}$ - phương sai của $Z$ và là giá trị hữu hạn. Giá trị thông số tính toán $Z_{0}^{*}$ tại điểm 0 được xác định theo các công thức sau:

$$
Z_{0}^{*}=m+\sum_{i=1}^{n} \lambda{ }_{0}^{i}\left(Z_{i}-m\right)
$$

và phương sai của hiệu giữa giá trị tính toán và giá trị thực của thông số (giá trị thực của thông số không biết được) được xác định như sau:

$$
\begin{aligned}
& \operatorname{var}\left(Z_{0}^{*}-Z_{0}\right)=\operatorname{var}(Z)-\sum_{i=1}^{n}\left[\beta_{0}^{i} C\right. \\
& \left.\left(\mathbf{x}_{i}-\mathbf{x}_{0}\right)\right]=\sigma_{Z}^{2}-\sum_{i=1}^{n} \beta_{0}^{i} C\left(\mathbf{x}_{i}-\mathbf{x}_{0}\right)
\end{aligned}
$$

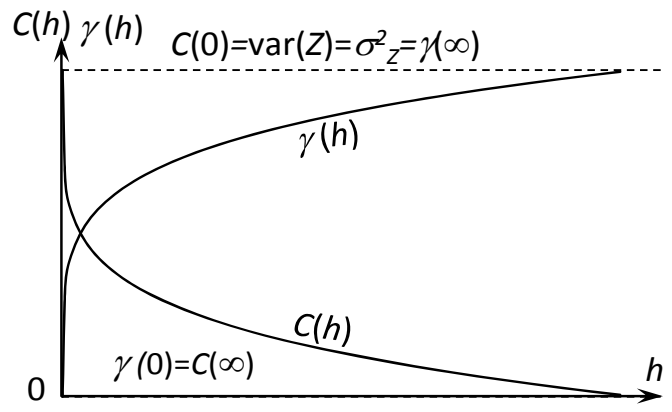

Hình 2. Hàm hiệp phương sai và Variogam

Các hệ số trọng số $\beta_{0}^{i}$ được xác định bằng cách giải hệ $n$ phương trình tuyến tính sau: 
Tạp chí Các Khoa học về Trái Đất, 38 (1), 79-89

$$
\begin{aligned}
& \sum_{j=1}^{n} \beta{ }_{0}^{j} C\left(\mathbf{x}_{i}-\mathbf{x}_{j}\right)=C\left(\mathbf{x}_{i}-\mathbf{x}_{0}\right), \\
& i=1,2, \ldots, n
\end{aligned}
$$

\subsubsection{Giả thiết nội tại}

Trong trường hợp này, giả sử rằng thậm chí phương sai của biến số $Z$ không hữu hạn, nhưng phương sai của số gia bậc nhất của $Z$ hữu hạn và dừng bậc hai, tức là thỏa mãn:

$$
\left.\begin{array}{c}
E[Z(\mathbf{x}+\mathbf{h})]-E[Z(\mathbf{x})]=m(\mathbf{h}) \\
\operatorname{var}[Z(\mathbf{x}+\mathbf{h})-Z(\mathbf{x})]=2 \gamma(\mathbf{h})
\end{array}\right\}
$$

trong đó: $\mathbf{h}$ - vectơ trong không gian 1, 2 hoặc 3 chiều, $\chi(\mathbf{h})$ - hàm phụ thuộc vào khoảng cách $\mathbf{h}$ được gọi là "Variogram" và được xác định như sau:

$$
\begin{aligned}
& \gamma(\mathbf{h})=\frac{1}{2} \operatorname{var}[Z(\mathbf{x}+\mathbf{h})-Z(\mathbf{x})] \\
& =\frac{1}{2} E\left\{[Z(\mathbf{x}+\mathbf{h})-Z(\mathbf{x})]^{2}\right\}
\end{aligned}
$$

Giá trị biến thông số $Z$ được xác định theo công thức trọng số sau:

$$
Z_{0}^{*}=Z^{*}\left(\mathbf{x}_{0}\right)=\sum_{i=1}^{n} \beta_{0}^{i} Z_{i}
$$

và các hệ số trọng số xác định qua việc giải hệ $n+1$ phương trình sau:

$$
\begin{aligned}
& \sum_{j=1}^{n} \beta_{0}^{j} \gamma\left(\mathbf{x}_{i}-\mathbf{x}_{j}\right)+\mu=\gamma\left(\mathbf{x}_{i}-\mathbf{x}_{0}\right), \\
& i=1,2 \ldots, n ; \quad \sum_{i=1}^{n} \beta_{0}^{i}=1
\end{aligned}
$$

Phương sai của sai số tính là:

$$
\operatorname{var}\left(Z_{0}^{*}-Z_{0}\right)=\sum_{\mathrm{i}=1}^{\mathrm{n}} \beta_{0}^{\mathrm{i}} \gamma\left(\boldsymbol{x}_{i}-\boldsymbol{x}_{0}\right)+\mu
$$

Chúng ta có thể nhận thấy rằng Kriging cho giá trị tính toán tại điểm đo thực tế bằng chính giá trị đo được. Giá trị biến ngẫu nhiên $Z_{0}$ với $95 \%$ độ tin cậy là:

$$
\begin{aligned}
& Z_{0}^{*}=\sum_{i=1}^{n} \beta{ }_{0}^{i} Z_{i} \pm 2 \sigma \\
& =\sum_{i=1}^{n} \beta{ }_{0}^{i} Z_{i} \pm 2 \sqrt{\operatorname{var}\left(Z_{0}^{*}-Z_{0}\right)}
\end{aligned}
$$

trong đó: $\sigma$ - độ lệch chuẩn.

\subsubsection{Các dạng Variogram}

Như Variogram được xác định theo công thức (10), các giá trị Variogram có thể tính từ các số đo thông số tại các vị trí $i$, sau đó dạng Variogram có thể xác định bằng cách so sánh hình dáng Variogram thực tế với các dạng Variogram chuẩn. Thông thường có 6 dạng Variogram chính (Ghislain de Marsily, 1987; Nguyễn Văn Hoàng, 1998). Trong thực tiễn từ những năm 1990 đến nay khi công nghệ GIS phân tích không gian phát triển mạnh, đã có thêm một số dạng Variogram được đề xuất. Tuy nhiên trong lĩnh vực ĐCTV các thông số liên quan thông thường tuân thủ 6 dạng chính (Ghislain de Marsily, 1987; Nguyễn Văn Hoàng, 1998).

Áp dụng phương pháp luận nêu trên, Nguyễn Văn Hoàng đã trình bày kết quả ứng dụng phương pháp Địa Kriging đối với hàm lượng sắt trong nước và hệ số dẫn nước tầng Pleistocen khu vực Hà Nội (Nguyễn Văn Hoàng, 1998).

\subsubsection{Giả thiết không dìng}

Trong trường hợp này kỳ vọng toán học $E[Z(\mathbf{x})]=m(\mathbf{x})$ của biến ngẫu nhiên $Z$ chưa biết và không phải là hằng số nên không thể xác định Variogram từ số liệu thí nghiệm được. Variogram là hàm số không chỉ của khoảng cách mà còn cả hướng vì kỳ vọng toán học của biến thay đổi theo hướng. Để có thể vẫn tiến hành Kriging theo hai trường hợp đầu, có thể tiến hành đơn giản hóa như sau: (i) Giả sử $Z$ dừng trong một diện cục bộ nào đó; (ii) Giả sử biết được kỳ vọng toán học $m(\mathbf{x})$, và (iii) Giả sử Variogram $\gamma$ dừng và đã biết.

\section{Kết quả và thảo luận}

\subsection{Kết quả xác định dạng Variogram thể hiện quy luật phân bố độ tổng khoáng hóa}

Số điểm mẫu độ tổng khoáng hóa TCN qh là 50 (trung bình 18,46 km²/1điểm), TCN qp2 là 181 điểm (trung bình $5,10 \mathrm{~km}^{2} / 1$ điểm) và $\mathrm{TCN}$ qp1 là 106 điểm (trung bình $8,71 \mathrm{~km}^{2} / 1$ điểm). Đã xác định được giá trị Variogram $M$ theo không gian và phân tích xác định dạng Variogram phù hợp nhất từ 06 dạng nêu trên. Kết quả cho thấy dạng phù hợp nhất là dạng lũy thừa với số mũ $<1$ và bình phương hệ số tương quan của đường Variogram với các giá trị 


\section{N.Đ. Rỡi/Tạp chí Các Khoa học về Trái Đất, Tập 38 (2016)}

Variogram kinh nghiệm rất lớn từ 0,859 (TCN qp1) đến 0,906 (TCN qp2) (các hình 3-5).

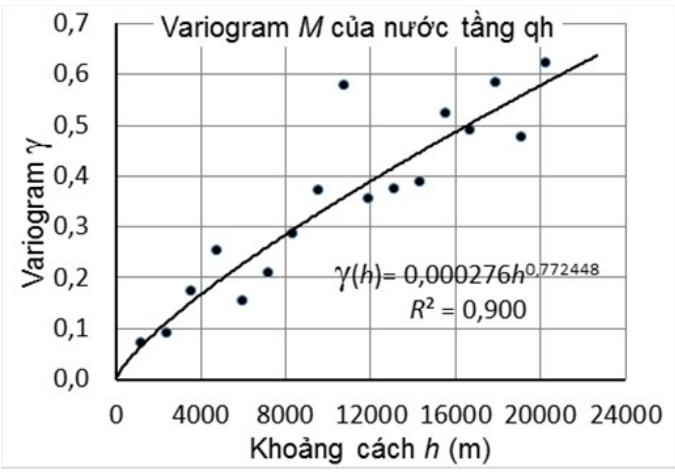

Hình 3. Variogram $M$ của nước TCN qh

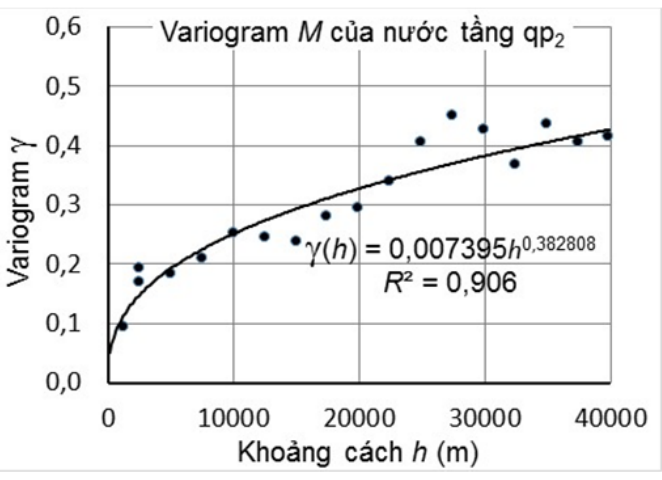

Hình 4. Variogram $M$ của nước TCN qp2

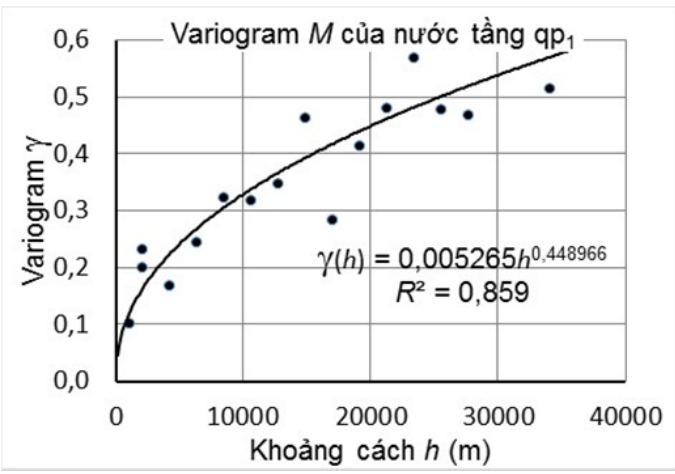

Hình 5. Variogram $M$ của nước TCN qp1

Sử dụng các phương trình Variogram được xác định này cùng các điểm kết quả phân tích $M$ đã tiến hành nội suy độ tổng khoáng hóa đối với toàn khu vực nghiên cứu. Lưới các điểm nội suy độ tổng khoáng hóa là $500 \mathrm{~m} \times 500 \mathrm{~m}$. Hai thuật toán nội suy theo phương pháp Kriging là: số điểm số liệu sử dụng để nội suy là 6 và Kriging trượt bán kính đường tròn chứa các điểm sử dụng để nội suy không lớn hơn $23 \mathrm{~km}$ (đối với $\mathrm{TCN}$ qh) và $34 \mathrm{~km}$ (đối với TCN qp2 và $\mathrm{TCN}$ qp1) được xác định qua khoảng cách lớn nhất trên các đồ thị Variogram. Thực tế tính toán giá trị trung bình khoảng cách từ điểm tính nội suy đến các điểm sử dụng tính toán nhỏ hơn nhiều các giá trị này.

\subsection{Kết quả phân vùng chất lựng ND円 theo độ tổng khoáng hóa}

Sau khi xác định được $M$ nội suy trên toàn khu vực nghiên cứu, đã tiến hành phân vùng chất lượng NDĐ của các TCN theo giá trị $M$ trên cơ sở Thông tư số 09/2014/TT-BTNMT ngày 17/02/2014 của Bộ Tài nguyên và Môi trường quy định về kỹ thuật lập bản đồ chất lượng NDĐ (Bộ TN\&MT, 2014).

Khu vực nghiên cứu hầu như không có NDĐ thuộc loại mặn (chỉ có 1 mẫu TCN qp2 và 1 mẫu TCN qp1 có $M$ cao hơn $3 \mathrm{~g} / 1$ không đáng kể), chủ yếu chỉ tới loại nước lợ. Kết quả phân vùng NDĐ đối với từng $\mathrm{TCN}$ thể hiện trong 3 sơ đồ (hình 68). Ngoài ra cũng xây dựng sơ đồ phân bố nước lợ của $03 \mathrm{TCN}$ để thể hiện diện phân bố và đánh giá mối quan hệ giữa chúng; đồng thời cũng thể hiện các công trình khai thác NDĐ tập trung trong khu vực nhằm đánh giá nguy cơ xâm nhập nước lợ và nước mặn vào các công trình khai thác nước.

\subsection{Về hiện trạng khai thác NDĐ}

Trên địa bàn tỉnh Hưng Yên hiện có nhiều công trình khai thác NDĐ đang hoạt động cung cấp nước sản xuất, sinh hoạt cho các $\mathrm{KCN}$, các khu đô thị và các khu vực nông thôn (Sở Tài nguyên và Môi trường Hưng Yên, 2014): (i) Các trạm cấp nước cho các $\mathrm{KCN}$ có tổng công suất là $~ 81.080$ $\mathrm{m}^{3} / \mathrm{ng}$; (ii) Các trạm cấp nước cho các khu đô thị có tổng công suất là $13.500 \mathrm{~m}^{3} / \mathrm{ng}$; (iii) Có 9 trạm cấp nước nông thôn khai thác NDĐ với công suất $\sim 7.060 \mathrm{~m}^{3} / \mathrm{ng}$; (iv) Toàn tỉnh có khoảng 145.400 giếng khoan dạng "Unicef" trong các TCN Pleistocen với tổng lưu lượng nước khai thác trung bình ước khoảng $145.400 \mathrm{~m}^{3} / \mathrm{ng}$.

Như vậy, tổng lưu lượng khai thác NDĐ toàn tỉnh Hưng Yên là khoảng $247.042 \mathrm{~m}^{3} / \mathrm{ng}$. Hầu hết các lỗ khoan khai thác NDĐ phục vụ sản xuất và 
Tạp chí Các Khoa học về Trái Đất, 38 (1), 79-89

sinh hoạt của các $\mathrm{KCN}$, các khu đô thị và cấp nước nông thôn khai thác từ $\mathrm{TCN}$ qp1 (125 công trình được cấp phép khai thác từ TCN qp1 và 23 công trình được cấp phép khai thác từ TCN qp2) (hình 9). Các giếng khai thác phân bố phân tán, tập trung nhiều nhất ở khu vực huyện Văn Lâm, Văn Giang,
Mỹ Hào và Yên Mỹ. Do các giếng dạng "Unicef" khai thác nhỏ lẻ phân bố đều khắp, nên trên phễu hạ thấp mực nước toàn khu vực có các phễu cục bộ tạo bởi các công trình khai thác nước tập trung. Dạng phễu hạ thấp mực nước tương ứng với lưu lượng khai thác được thể hiện trên hình 9.



Hình 6. Phân vùng NDĐ theo $M$ của TCN qh 
N.Đ. Rỡi/Tạp chí Các Khoa học về Trái Đất, Tập 38 (2016)

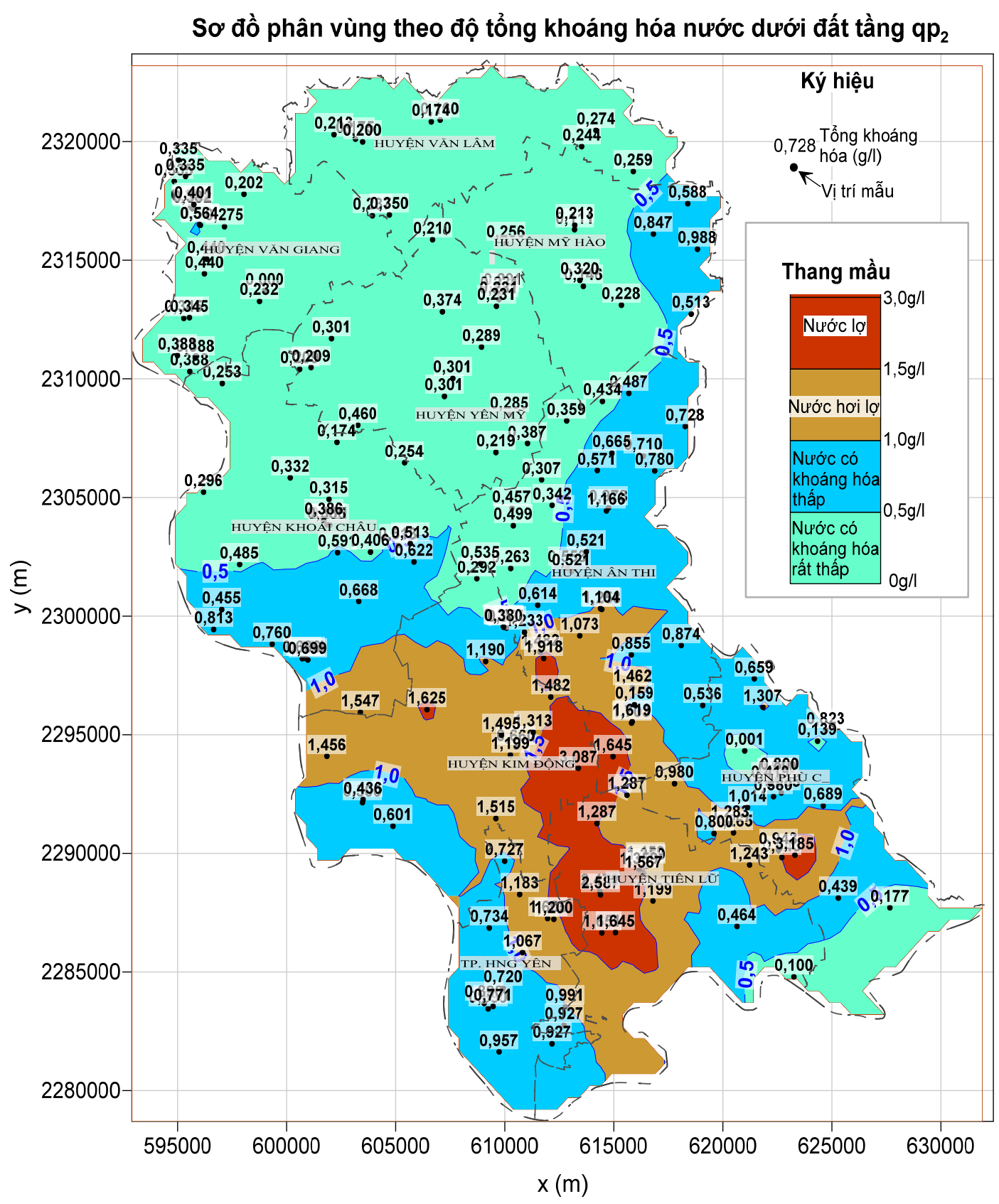

Hình 7. Phân vùng NDĐ theo $M$ của TCN qp2 
Tạp chí Các Khoa học về Trái Đất, 38 (1), 79-89

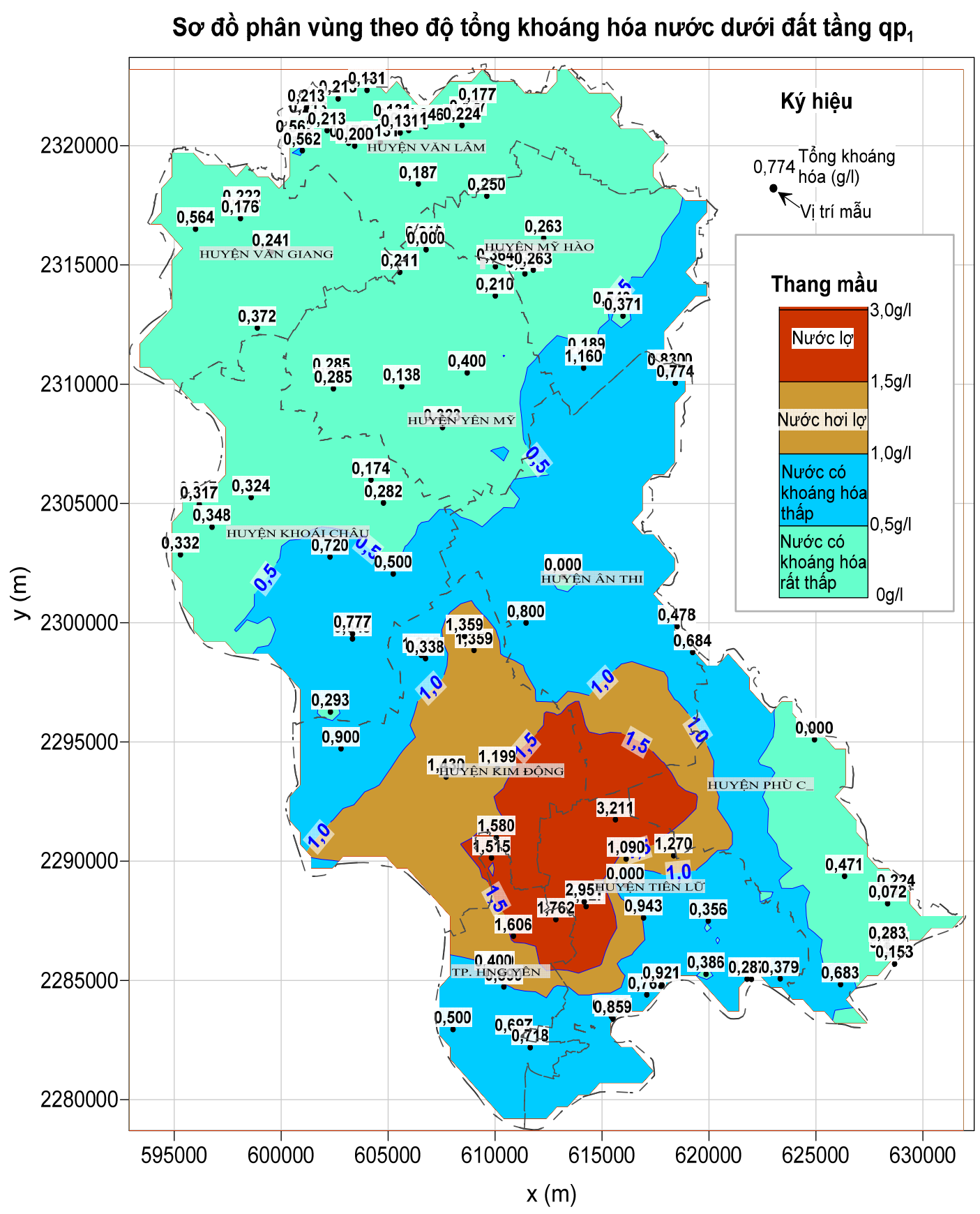

Hình 8. Phân vùng NDĐ theo $M$ của TCN qp1 
N.Đ. Rỡi/Tạp chí Các Khoa học về Trái Đất, Tập 38 (2016)

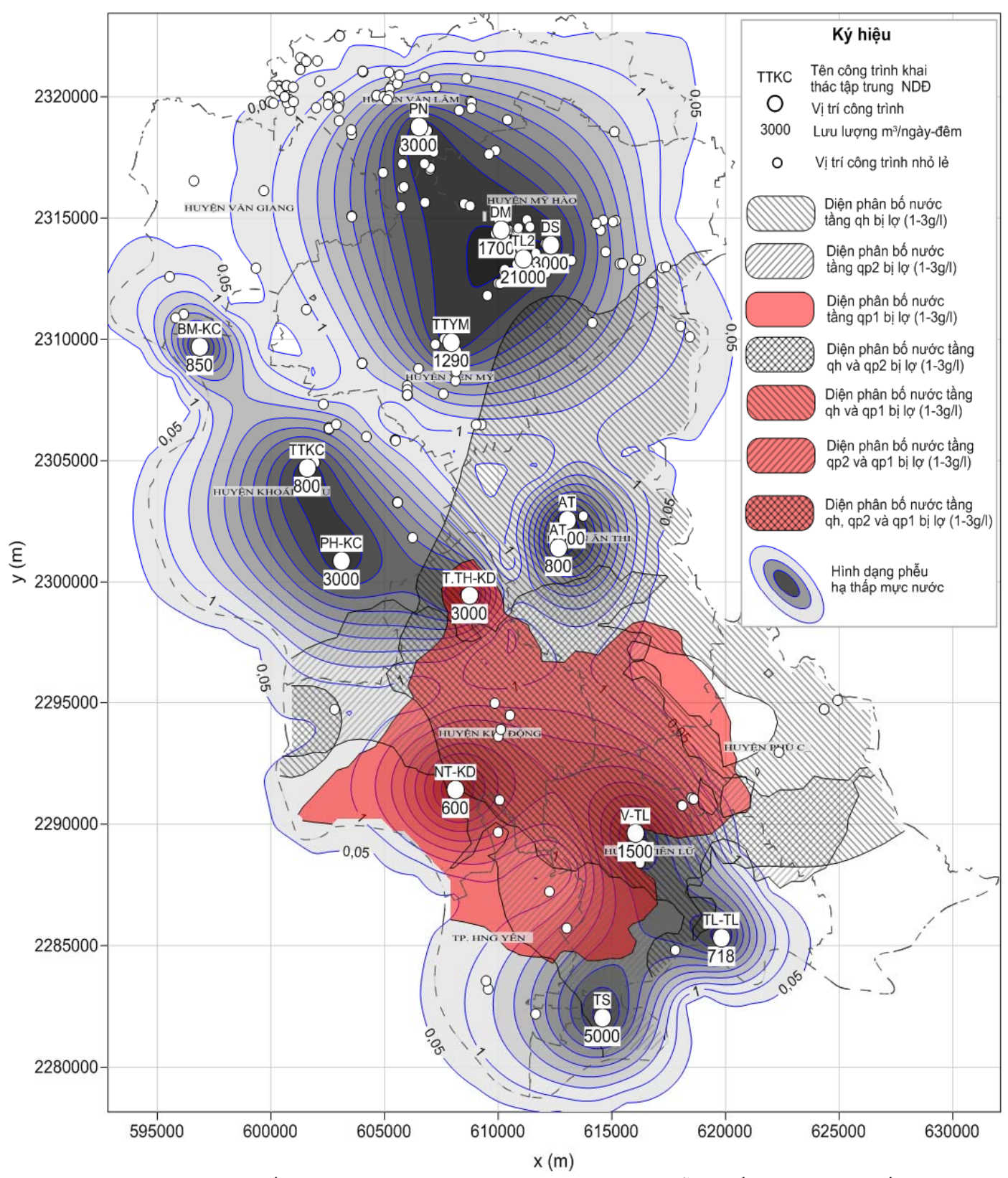

Hình 9. Phân vùng chất lượng NDĐ theo $M$, các công trình khai thác, dạng phễu hạ thấp mực nước dưới đất

\section{Nhận xét - kết luận}

Từ kết quả phân vùng độ tổng khoáng hóa NDĐ nêu trên, có thể nhận thấy một số quy luật phân bố nhu sau:

- Độ tổng khoáng hóa của cả $3 \mathrm{TCN}$ trong khu vực nghiên cứu có phân bố không gian theo hàm lũy thừa với giá trị số lũy thừa nhỏ hơn 1 . Hệ số tương quan giữa hàm Variogram và các giá trị Variogram thực nghiệm có giá trị rất cao cho thấy mức độ tin cậy cao của qui luật phân bố được lựa chọn này; 
Tạp chí Các Khoa học về Trái Đất, 38 (1), 79-89

- Trong khu vực nghiên cứu, NDĐ của cả 3 TCN chủ yếu thuộc loại nước lợ (tầng qp2 chỉ có 1 mẫu có $\mathrm{TDS}=3,087 \mathrm{~g} / 1$, tầng qp1 chỉ có 1 mẫu có $\mathrm{TDS}=3,211 \mathrm{~g} / \mathrm{l})$. Diện tích có độ tổng khoáng hóa trong khoảng $1 \div 3 \mathrm{~g} / 1$ (từ hơi lợ đến lợ) như sau: (i) TCN qh là $\sim 310 \mathrm{~km}^{2}$ (chiếm $\sim 33 \%$ diện tích); (ii) TCN qp2 khoảng $210 \mathrm{~km}^{2}$ (chiếm $\sim 23 \%$ diện tích); (iii) TCN qp1 khoảng $180 \mathrm{~km}^{2}$ (chiếm $20 \%$ diện tích);

- Diện tích phân bố nước lợ trong TCN qh và $\mathrm{TCN}$ qp2 rất khác nhau chứng tỏ $2 \mathrm{TCN}$ này có sự xáo trộn theo phương thẳng đứng rất không đáng kể hoặc không có sự xáo trộn. Điều này bổ sung cho kết luận của nhóm tác giả Nguyễn Văn Hoàng rằng 2 TCN này hầu như không có quan hệ thủy lực do sự có mặt của lớp thấm nước yếu giữa 2 tầng (Nguyễn Văn Hoàng và nnk, 2015);

- Diện tích phân bố nước lợ trong TCN qp2 và TCN qp1 tương đối trùng nhau, chỉ có hình dáng phân bố nồng độ hơi khác nhau, có nghĩa là sự phân bố lợ - mặn của $2 \mathrm{TCN}$ này tương đồng nhau, do: (i) Sự trùng hợp ngẫu nhiên của diện lợ - mặn của 2 TCN này; (ii) Có sự xáo trộn theo phương thẳng đứng qua cửa sổ ĐCTV (các lỗ khoan tại khu vực này cho thấy lớp thấm nước yếu giữa chúng rất mỏng hoặc không có).

Kết hợp kết quả phân vùng độ tổng khoáng hóa NDĐ với hiện trạng khai thác và hình dạng phễu hạ thấp mực NDĐ đưa ra một số thảo luận và có thể đề xuất một số giải pháp sau (có thể tiến hành độc lập hoặc đồng thời):

- Trong khu vực phân bố NDĐ bị lợ, số lượng các công trình khai thác ít, tuy nhiên vẫn có một số công trình khai thác từ $\mathrm{TCN}$ qp2 và $\mathrm{TCN}$ qp1 với công suất lớn do nhu cầu cần thiết phải khai thác sử dụng mà không có nguồn nước thay thế;

- Có một số phễu hạ thấp mực nước cục bộ bao quanh các công trình khai thác nước tập trung với công suất lớn nằm ở rìa các khoảnh nước lợ của TCN qp1 và TCN qp2. Vì vậy nguy cơ mở rộng ranh giới nước lợ sang các khu vực NDĐ nhạt là không thể tránh khỏi;

- Bổ sung nước nhạt (nước mặt, nước mưa) vào các khoảnh NDĐ bị lợ;

- Khai thác hết thể tích NDĐ bị lợ để các diện tích này được thay thế bằng nước nhạt;
- Dọc ranh giới nước nhạt - lợ xây dựng hệ thống các lỗ khoan ép nước nhạt nhằm tạo mặt phân thủy và bổ cập nhân tạo;

- Dọc ranh giới nước nhạt - lợ, phía trong phần nước lợ xây dựng hệ thống các lỗ khoan khai thác NDĐ bị lợ nhằm không cho nước lợ - mặn xâm nhập khu vực NDĐ nhạt tạo mặt phân thủy và bổ cập nhân tạo.

\section{Tài liệu dẫn}

Nguyễn Văn Độ (chủ biên), 2002: Đo vẽ lập bản đồ địa chất, địa chất thủy văn - địa chất công trình tỷ lệ 1:50.000 vùng Hưng Yên - Phủ Lý. Lưu trữ Liên đoàn Quy hoạch và Điều tra Tài nguyên nước Miền Bắc, 180tr.

Ghislain de Marsily, 1987: Quantitative Hydro-geology: Groundwater Hydrology for Engineers. Academic Press Inc., Harcourt Brace Jovanovich Publishers, pp.284-329.

Nguyễn Văn Hoàng, 1998: Phương pháp Kriging và ứng dụng nó để tính hệ số dẫn áp và nồng độ sắt trong nước dưới đất vùng Hà Nội. Tuyển tập các báo cáo khoa học: Hội nghị khoa học lần thứ 13-Trường Đại học Mỏ-Địa chất; Quyển 3, tr.144-149.

Nguyễn Văn Hoàng, Nguyễn Đức Rỡi, 2015: Study on the hydraulic connectivity between Holocene and Pleistocene aquifers and the Red river in Hung Yen city area. Tạp chí Khoa học Đại học Quốc gia Hà Nội: Các Khoa học Trái đất và Môi trường; tập 31, số 2, pp.11-22.

Kemker Christine, 2014: Conductivity, Salinity and Total Dissolved Solids. Fundamentals of Environmental Measurements. Fondriest Environmental, Inc. 3 Mar 2014.

Phạm Quý Nhân (chủ biên), 2007: Báo cáo kết quả thăm dò đánh giá trữ lượng khai thác NDĐ Trung tâm dệt kim Phố Nối công suất $5.000 \mathrm{~m}^{3} / \mathrm{ng}$. Lưu trữ Sở TN\&MT, Hưng Yên, 135tr.

Bộ Tài nguyên và Môi trường, 2008: QCVN 09: 2008/BTNMT- Quy chuẩn kỹ thuật Quốc gia về chất lượng nước ngầm. Lưu trữ Bộ TN\&MT, 6tr.

Bộ Tài nguyên và Môi trường, 2014: Thông tư số 09/2014/TTBTNMT: Quy định kỹ thuật lập bản đồ chất lượng nước dưới đất tỷ lệ 1:50.000. Lưu trữ Bộ TN\&MT, 6tr.

Sở Tài nguyên và Môi trường Hưng Yên, 2014: Báo cáo tình hình khai thác, sử dụng nước tỉnh Hưng Yên năm 2014. Lưu trữ Sở TN\&MT, Hưng Yên, 14tr. 
N.Đ. Rỡi/Tạp chí Các Khoa học về Trái Đất, Tập 38 (2016) 